SPATIAL COGNITION AND COMPUTATION, vol(issue), 1-end

Copyright (C) YYYY, Lawrence Erlbaum Associates, Inc.

\title{
Route Aware Maps: Multigranular Wayfinding Assistance
}

\author{
Falko Schmid \\ Kai-Florian Richter \\ Denise Peters \\ SFB/TR 8 Spatial Cognition, \\ University of Bremen, Germany
}

In unfamiliar environments, people need assistance to find their way. One predominant form of such assistance is maps. In constructing these maps, there is a conflict between concentrating on the essential information for wayfinding, namely the route, and providing overview information of the environment. The former eases information extraction by reducing visual clutter, the latter allows for reorientation in the environment even if the route has been left. In this paper we present route aware maps, an approach that combines the best of both (map) worlds. We argue how route information can be embedded in its surrounding environment, i.e., the global spatial context, without introducing unnecessary visual clutter. We present a construction process that results in route aware maps and detail each step of this process. Route aware maps shall ease information extraction by focusing on the route as the crucial piece of information and at the same time impart the feeling of efficient and safe navigation by keeping the wayfinder in global context. Providing a global context in route following invokes spatial awareness with respect to the overall environment and, thus, decreases the (felt) risks of making wayfinding errors.

Keywords: wayfinding assistance, schematic maps, spatial context, key hole problem

Correspondence concerning this article should be addressed to Falko Schmid SFB/TR 8 Spatial Cognition, P.O. Box 330 440, 28334 Bremen schmid@sfbtr8.uni-bremen.de 


\section{SCHMID, RICHTER, PETERS}

\section{Introduction}

To successfully navigate, wayfinders need to know which directions to turn to at the crucial spots along their way, namely the decision points. If the environment is unfamiliar, wayfinders need assistance in taking these decisions. This assistance needs to communicate all information that is necessary to reliably enable wayfinders to perform the right actions, but should refrain from providing any excess information as this aggravates correctly interpreting the assistance (cf. Schneider \& Taylor, 1999; Baus et al., 2002; Richter \& Klippel, 2005).

Likewise, there is evidence that assistance is most efficient if wayfinders feel confident about taking the correct decisions (Ross et al., 2004; Ishikawa et al., 2008). An important step in evoking this confidence is to keep a wayfinder in context. Being in context here refers to wayfinders knowing (approximately) where in an environment they are, i.e., to allowing for global orientation. Such global orientation invokes a "sense of place" with respect to the environment at hand. Knowing where you are in an environment allows for reorientation after a wayfinding error, which is crucial for the confidence of being able to find the way successfully.

In this paper, we present route aware maps, an approach to map-based assistance that on the one hand focuses on the essential information for wayfinding, namely the route, and on the other hand evokes a sense of place by intelligently embedding this route in its global context. The paper is structured as follows: in the next section, we will provide some more background on the conflict of focusing on essential information and keeping users in context by discussing the properties of different types of maps. Section 3 then introduces the construction process for route aware maps in detail. Here, we explicate the individual building blocks we combine to create route aware maps. Each building block in itself helps to embed the provided information in a global context. Section 4 discusses route aware maps in light of other approaches to graphical wayfinding assistance. Section 5, finally, concludes the paper with an outlook on future work.

\section{Wayfinding and Maps}

Wayfinding is a daily routine for people. It is a purposive, directed, motivated activity to follow a route from origin to destination (Golledge, 1999). According to Montello (2005), it reflects the cognitive processes going on during navigation, as opposed to locomotion, which covers the activities of the sensory and motor system. A route is a behavioral pattern describing the way someone takes from an origin to a destination; it covers a directed movement activity (Klippel, 2003; Montello, 2005). This is opposed to a path, which corresponds to physical entities, i.e., the segments of a network of ways, that movement has been performed on.

Wayfinding requires attention in order to be successfully performed. Still- 
ROUTE AWARE MAPS 3

like for many daily routines-for frequently recurring ways, such as the way from your home to your work, the required level of attention will be low. No assistance is needed to reach your destination. In unknown environments, however, the required attention is high. And usually assistance is needed to successfully find your way in these environments. Such assistance is typically provided by verbal or graphical means, i.e., either as (spoken or written) text or as maps. The crucial information that needs to be communicated with this assistance is what to do at those places where there is more than one possible continuation of the route, i.e., at the decision points (Daniel \& Denis, 1998). In this paper, we focus on maps as wayfinding assistance. Broadly, we can distinguish two types of maps: 1) strip-like maps that only present the route to take; 2) survey maps that show an overview of the environment (cf. also Freksa, 1999).

The first type of maps provides the smallest possible set of complete route information that is needed to find your way, i.e., that is required to (re-)orient along the route. These maps only depict the route with an indication of where the decision points are along that route. Accordingly, they have very little visual clutter as they depict only the route. However, in case a wayfinder deviates from the depicted route by mistake, there is no information available to reorient as there is no information provided that relates the route to its surrounding environment. Therefore, once off the route, the wayfinder is lost. In terms of spatial awareness, these maps provide knowledge on location only locally with respect to the route, but not globally with respect to the overall environment. Essentially, today's mobile navigation systems work the same way. They provide information on the next action to be performed when it is due; they hardly provide any overview information that would allow users to reorient themselves more globally. Of course, mobile navigation systems still provide information when the intended route has been left by re-calculating a route and starting again.

These arguments mostly also hold for sketch maps as they are often provided by people to indicate which way to go. These maps usually depict some salient features encountered along the way additionally to the route (cf. Tversky \& Lee, 1998, 1999). But they still do not allow for a more global orientation as the embedding of the route in the environment is largely missing.

Secondly, there is the classical city map, which is nowadays often replaced by an electronic map extracted from the Internet. These maps show an overview of an environment by depicting information evenly distributed across the selected area. Accordingly, such maps contain a lot of excess information and, thus, visual clutter. Extracting, understanding and keeping track of the route to take is cognitively demanding (cf. Phillips, 1979; Rosenholtz et al., 2007). In principle, by displaying the route as it is embedded in its environment, these maps allow for communicating a sense of place, i.e., they provide global knowledge on one's location. However, this is masked by having to deal with a lot of unnecessary information. 


\section{SCHMID, RICHTER, PETERS}

In the following, we detail an approach to combining the best of both (map) worlds. We present route aware maps (RAMs) that concentrate on the route as the essential information to reach a destination, but also depict the information needed to anchor the route within its relevant spatial and functional context. Our aim is to develop a map, which highlights the route but still allows for (global) reorientation on different levels of granularity in case a wayfinder loses track and strays off the route. This map is schematic (Klippel et al., 2005) in that it focuses on the relevant information for successful route following. This goes beyond merely depicting only the route, but disregards excess information as it is provided by survey maps. We believe that RAMs provide a wayfinder with a sense of place by using a routespecific embedding of the essential wayfinding information in a global context. The elements selected for depiction shall impart the feeling of efficient and safe navigation.

\section{Route Aware Maps}

RAMs combine the concept of strip maps with means that allow for a wayfinder keeping a global orientation and recovering from wayfinding errors. Therefore, they increase a wayfinder's confidence during navigation. By embedding the route in its spatial context on different levels of granularity, we create assistance which allows for (approximate) navigation towards the goal even if a wayfinder makes errors. Due to the information presented additionally to the route we enable approximate localization and navigation, which lowers the burden on the wayfinder of being forced to take the right decision in order not to go astray (see Section 3.3.1 for more details).

RAMs can be used in both static as well as dynamic assistance. In a static scenario (e.g. print-out paper map) the information offered by RAMs is sufficient to allow wayfinders to follow a path and even make specific errors. In a dynamic scenario (e.g. GPS-assisted wayfinding), RAMs may serve as the means to communicate the spatial embedding of a route in the environment, especially on devices with small screens. These devices require a semantic selection of geographic entities in order to generate meaningful maps for the route and the current position of a wayfinder. RAMs provide mechanisms to detect and communicate the embedding context of the route, as well as the local context of wayfinders in their actual decision space to impart a sense of place on different levels of granularity. While in a static scenario RAMs would be produced once in advance of the trip, in a dynamic scenario they would be adaptive to the in-situ situation of network and traffic conditions, for example, and possibly changing requirements of the route to travel (e.g. due to an additional added target). In this article we focus on the generic generation process of RAMs and do not detail implications of a dynamization of RAMs, such as triggering recomputation, or adaptation of the visualization to changing positioning information. 
ROUTE AWARE MAPS 5

Construction of RAMs starts with the route itself. This route between origin and destination may be computed using any available metric, for example classical shortest paths (Dijkstra, 1959), or cognitively motivated approaches, such as the simplest path (Duckham \& Kulik, 2003), the most reliable path (Haque et al., 2007), or a path considering the corresponding simplest instructions (Richter \& Duckham, 2008). Then, stepwise we add additional information to the map that results in the embedding of the route in its spatial context. More specifically, we add information on:

- Initial and final orientation: are important for getting the wayfinder off in the right direction and for "homing" when close to the origin (Section 3.1);

- alternative routes: may allow a wayfinder to get back on track once accidentally having left the route (Section 3.2.3);

- Regions: provide an anchoring of the route in the environment (Section 3.3.1);

- Landmarks: help to disambiguate locations along the route and help to identify locations in the environment (Section 3.3.2).

In the following, we argue for adding these types of information to the basic route information and explain how it is done.

\subsection{Initial and Final Orientation}

Origin and destination of a route are crucial parts for successfully finding one's way (e.g., Michon \& Denis, 2001). At the origin, wayfinders need to initially orient themselves in order to get off in the right direction. The destination needs to be clearly identifiable in order to know that wayfinding has been successful. Further, especially in dense urban areas, such as a city center, there is an increased chance to miss crucial decisions and an increased need for reorientation near the destination. For example, this may be caused by a complex system of one-way streets or the need to find a parking space. Thus, it is sensible to not only guide wayfinders exactly to the destination location, but also to enable them to freely navigate in the nearby surroundings.

In order to support these two crucial processes, we display the environment around origin and destination in more detail. For the origin we extend the street network until no ambiguous situation is present any more: we expand the street network until each option the origin is reachable from is clarified by a defined configuration of streets (an identifiable intersection). Alternatively, if we identify a salient element (such as a park or a river) we employ the respective element instead to unambiguously identify this option. This first extension to the route allows a wayfinder for matching the spatial situations perceived in the environment around 


\section{SCHMID, RICHTER, PETERS}

origin and destination with that depicted on the map. For the destination region we expand the route similarly, but with a slightly higher scope (see Figure 1).

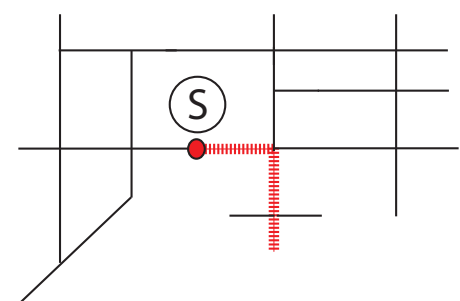

a)

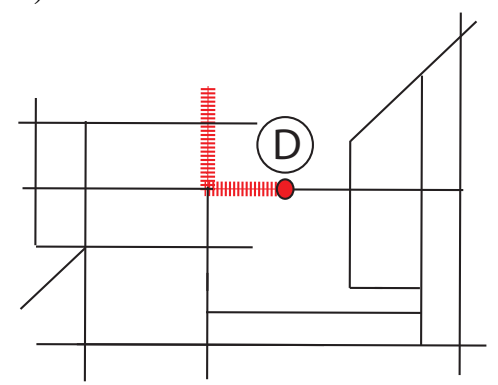

c)

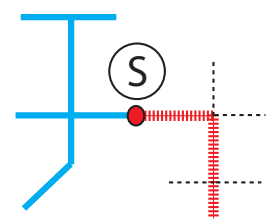

b)

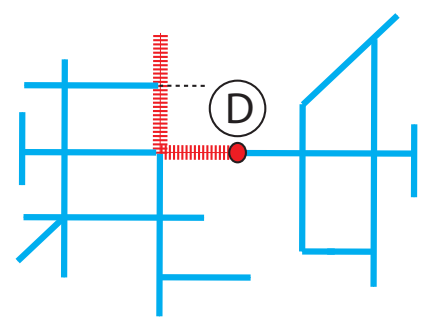

d)

Figure 1.: Initial and final orientation: a) Original initial surrounding, $\mathrm{S}$ is the starting point; the bold dashed line shows the route; b) Embedding of the starting point; bold solid lines shows the embedding area, thin dashed lines show intersections along the route; c) Original final surrounding, D is the destination; d) Embedding of destination (with depth 3).

More precisely, we use the following algorithm for adding information on origin and destination location:

1. We select all outgoing branches of the origin of the route and select all which are not member of the route.

2. We expand each selected branch until we either reach an intersection or a salient landmark. The corresponding streets are displayed on the map.

3. We select all outgoing branches of the destination.

4. Starting with each selected branch, we add all additional branches of the street network graph up until a configurable depth $k$ (without adding already existing branches again). The corresponding streets are displayed on the map. 


\subsection{Along the Route: Error Analysis and Alternative Routes}

As discussed earlier, wayfinding is a complex process in which errors easily occur due to manifold reasons. Errors may happen due to the wayfinder being inattentive, because the provided assistance (in form of a map) does not match with the encountered situation in the environment in the way it has been expected, or simply because the environmental situation itself is ambiguous and hard to understand. While it is impossible to predict every possible error that may occur during wayfinding, in RAMs we integrate information that allows recovery from two kinds of errors:

1. Local ambiguous or complex configuration of an intersection: based on capturing how humans conceptualize turns at intersections (e.g., Klippel, 2003; Haque et al., 2007), we can identify how many possible choices there are at an intersection and whether these choices potentially conflict with each other;

2. Global ambiguous situations can originate from monotone, recurrent, cueless environments as they often occur in modern suburbs, for example. Decision points, i.e., the relevant intersections, can be easily confused with other intersections due to the similarity in the environmental structure and the density of intersections.

The analysis performed to identify both kinds of errors is motivated by how humans conceptualize wayfinding situations and follows an information-theoretic approach. Local error analysis is explained next, global error analysis in Section 3.2.2. To enable wayfinders to recover from these errors, we introduce alternative routes as explained in Section 3.2.3.

\subsubsection{Local Error Analysis}

The first kind of error is tackled by analyzing the configuration of an intersection's branches. We employ a method we term Choreme Analysis (CA). CA is similar to the approach of identifying the most unambiguous instructions, introduced by Haque et al. (2007), but with a different resolution:

1. We discretize all angles formed by the incoming branch with all other branches based on the wayfinding choreme direction model as proposed in Klippel \& Montello (2007). As a result every angle is represented as a qualitative direction relation relative to the direction of travel.

2. According to this representation, we check if any two (or more) branches head in the same direction and hence are represented by the same relation. If this is the case and if they are relevant for the action to be performed at this intersection, a potential conflict is identified (cf. also Haque et al., 


\section{SCHMID, RICHTER, PETERS}

2007; Richter \& Klippel, 2005). A branch is relevant if its choreme representation corresponds to the direction to take at the intersection, i.e., if it is described by the same qualitative relation as the action to be performed at this intersection (e.g., "veer right"). Figure 2 illustrates such a conflict. Here, two outgoing branches of the intersection share the same direction concept.

3. We compute for every detected conflicting branch an alternative route to the destination (Section 3.2.3).

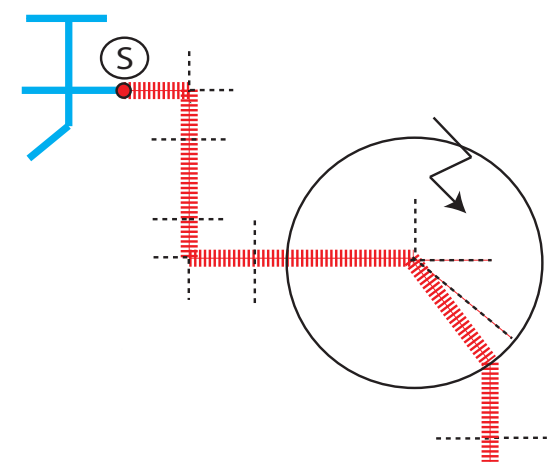

a)

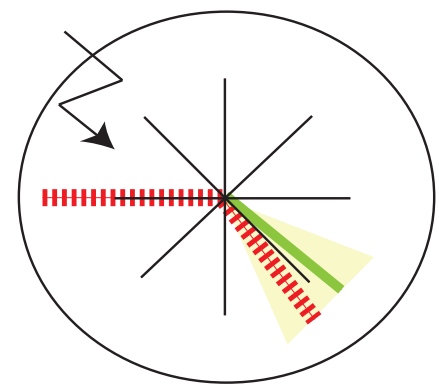

b)

Figure 2.: Local error analysis: a) Critical situation: two outgoing branches share the same direction concept, a conflicting situation is identified; b) In more detail: the Choreme Analysis.

\subsubsection{Global Error Analysis}

The second kind of errors, namely errors resulting from the recurrent, uniform structure of an environment is analyzed in a similar fashion as the first. The aim is to tackle two problems simultaneously. The first remains in the representation of spatial features in maps; the second in the structure of the environment. Automatically generated maps are always data-driven representations. They use cartographic symbols to visualize a selection of the spatial entities that exist in the real world. Maps are selective and limited in their visual language, a key concept differentiating them from satellite photos (e.g., MacEachren, 1995). For example, the two images in Figure 3 show a rather monotone environment in Chicago. When traveling along the east-west axis the streets have names and are not numbered. Traveling in such environments requires close attention to the perceived 


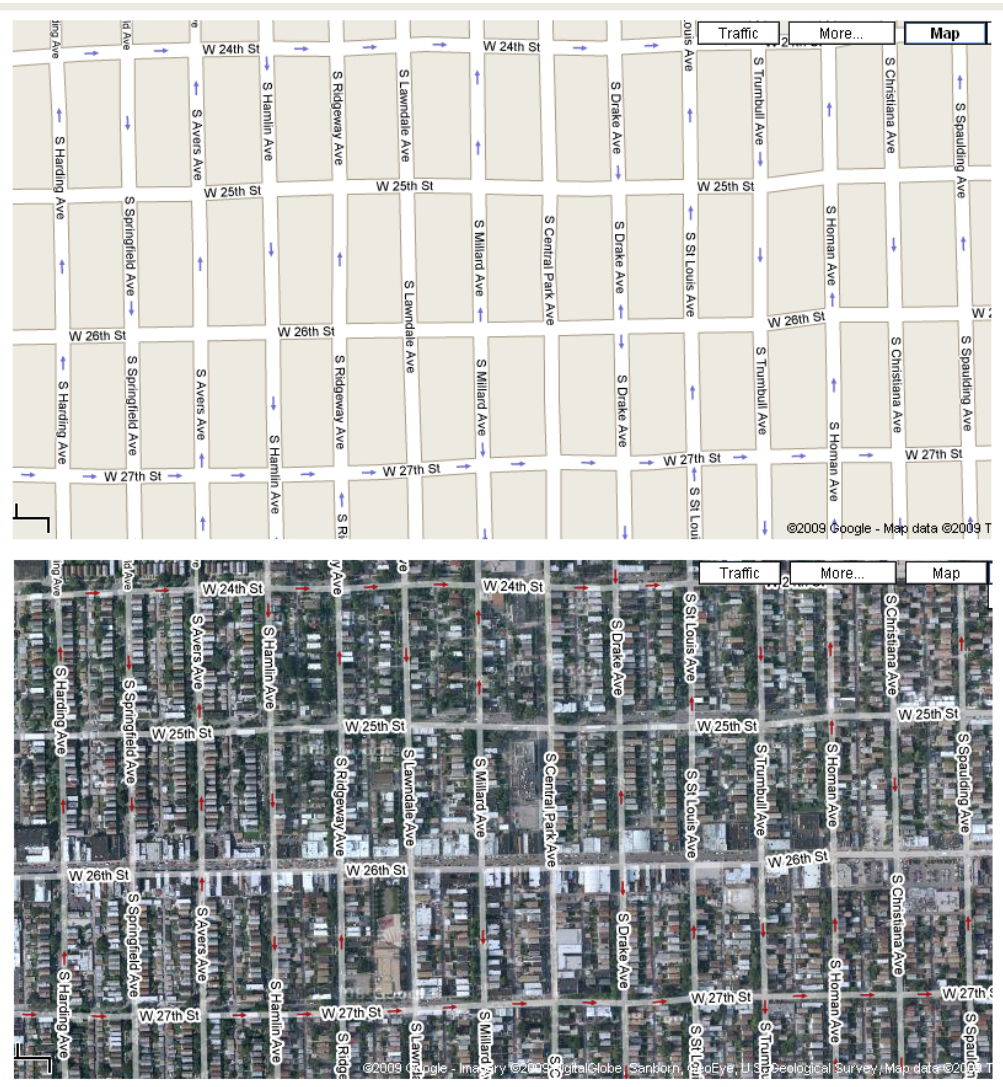

Figure 3.: A monotone environment in Chicago: at the top a map representation, at the bottom the corresponding satellite image. Images are taken from GoogleMaps.

environment in order to identify the correct decision point. Each intersection follows exactly the same conceptual configuration. There are no disambiguating elements, such as irregular structures or natural features, to segment the route beyond decision points. As Lynch (1960) pointed out, such monotone environments are hard to navigate.

We can operationalize uniformity in environmental structure as well as we can identify spatial particularities in order to support disambiguation. The algorithm works as follows and is illustrated in Figure 4:

1. We select a (configurable) number of intersections before and after the current decision point. "Before" and "after" are to be understood relative to the movement direction determined by the main route (cf. Richter \& Klippel, 
10 SCHMID, RICHTER, PETERS

2007). In our current implementation, we select one intersection before and one after the decision point, even though this number may depend on the structure of the environment. Large grid environments, such as in Figure 3, may require a larger threshold than irregular environments (the analysis of the influence of an environment's structure on the required threshold is part of future work).

2. For all selected intersections, we calculate a qualitative representation of their configuration using a choreme encoding according to the $\mathrm{CA}$ as described above. However, here we are not interested in a single local configuration that may be conflicting, but in the similarity across the selected intersections' configurations.

(a) If the number of conceptual turns at two intersections is similar we mark the intersections as similar.

(b) If the similar intersections have a unique feature located at them, which is not present at the other intersections marked as similar, we select this feature to disambiguate the current intersection. Consequently, we remove the similarity mark for this intersection again, as it can be unambiguously identified in its local neighborhood by this local feature. Disambiguating features, such as parks or water bodies, must be perceptually unambiguous, i.e., the cartographic style used to represent them on the map must be perceptually different from the way all other features in the local surroundings are depicted.

3. If there still exist unresolved ambiguities, we compute an alternative route for those intersections that are marked as similar.

\subsubsection{Alternative Routes}

Alternative routes (ARs) are a key concept of RAMs. They are the link to embedding the main route from origin to destination in the street network. Whenever the (local or global) error analysis identifies a possible error source on the main route we compute an AR from the location of the possible error either back to the main route or directly to the destination. This allows for extracting only the part of the street network, which is structurally important for the main route. Within the alternative routes, we do not perform any error analysis. Cognitively, these routes define the relevant spatial context on a network level and serve as a fallback in case wayfinding errors have been made. Computationally, checking for errors on ARs as well would result in a recursive progression of potential errors that, in the worst case, would lead to displaying the complete street network and, thus, introducing unneeded visual clutter. 


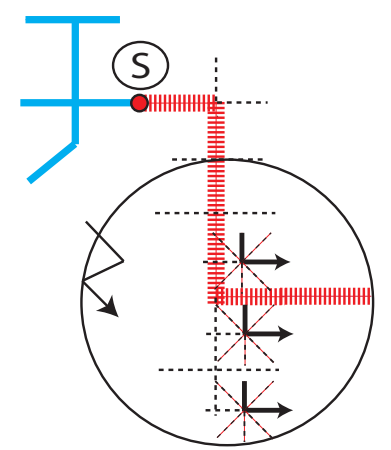

a)

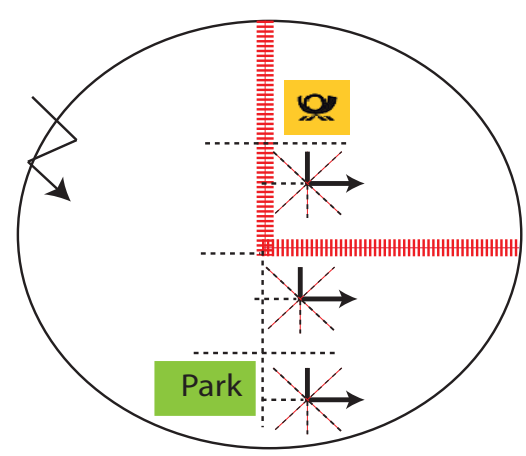

b)

Figure 4.: Global error analysis: a) Similarity analysis: the intersections before and after the decision point are identified as being similar with respect to their configuration, a conflicting situation is identified; b) The situation is disambiguated by representing landmarks (post office and park).

Calculating ARs is based on the approach of "simplest paths" introduced by Duckham \& Kulik (2003). The AR algorithm works as follows and is illustrated in Figures 5 and 6:

1. We simulate a wayfinding error and "move" virtually off-route from the current decision point we identified as potential error source. In case of a local conflict, we move along a conflicting branch. In case of a global conflict, we take that branch at the conflicting intersection that corresponds to the branch we need to take at the actual decision point.

2. We block the branch we took. This will prevent the path search algorithm to simply compute the route back along this branch to the main route.

3. We compute the AR itself using the algorithm for "simplest paths." The algorithm penalizes nodes with many outgoing branches as they are deemed as complex; such nodes are mostly avoided. Therefore, in simplest paths competing branches hardly ever occur.

- In every step of the simplest path algorithm we check if the current selected node is either a node on the main route or corresponds to the destination itself. In either case we have found a valid AR and stop.

4. The AR just found is displayed on the map. 
12 SCHMID, RICHTER, PETERS

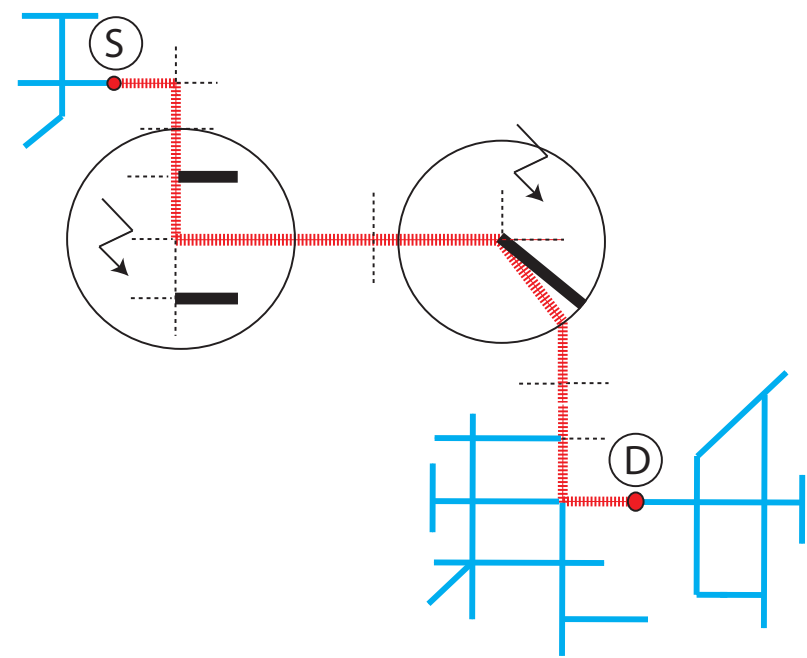

Figure 5.: Alternative routes: identifying all critical situations.

\subsection{Global and Local Orientation}

So far we have only dealt with local context, i.e., discussed information directly related to the route. However, with our aim of sparseness in providing information we need global spatial context as well to support successful wayfinding, especially when wayfinders get off the system of routes and need additional information to navigate to the destination. We need additional survey information in order to clarify the relations of the presented information (the main route and the ARs) to the actual environment wayfinding takes place in. We achieve this by introducing regions (Section 3.3.1) and landmarks (Section 3.3.2).

\subsubsection{Global Orientation: Regions}

In recent years, the impact of regions on human spatial conceptualization has been increasingly recognized. Wiener \& Mallot (2003) identified region-based navigation strategies. Their work emphasized the concept of a graph-like representation of spatial information in which representations of locations are interlinked without having exact metrical information. They introduced the hierarchical planning hypothesis which claims that people plan routes by using different levels of a hierarchical representation of space and also that regions are explicitly represented in spatial memory. Seifert introduced a hierarchical spatial planning approach based on regions as primitives (e.g., Seifert et al., 2007). Schmid (2008) developed an approach to generating maps based on individually known places and 


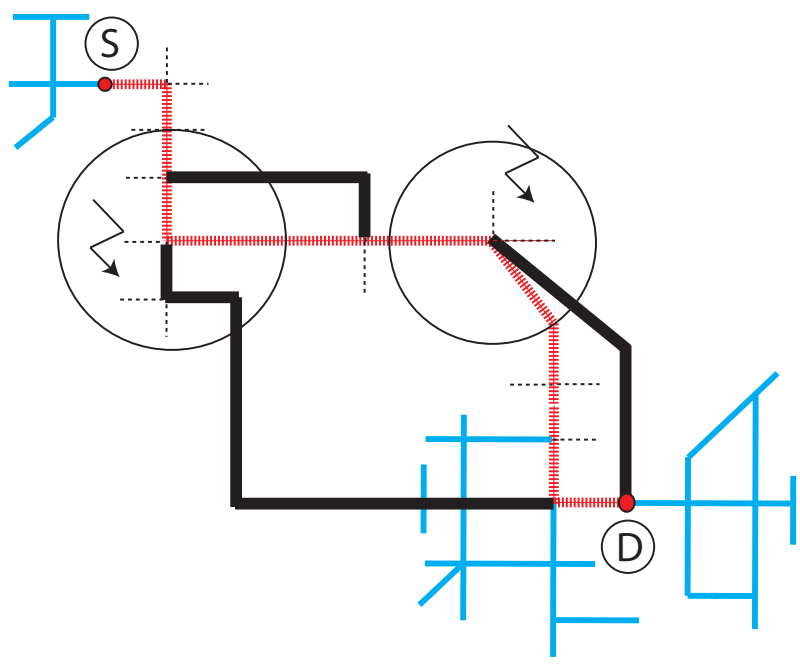

Figure 6.: Alternative routes: computing the ARs (bold solid lines) by employing the "simplest path" algorithm.

regions. Thus, just as landmarks (see below), regions are recognized as primitives in spatial orientation, navigation and communication.

In general, regions are hard to define as each individual seems to have a personal view of the concepts and borders of a region (e.g., Couclelis, 1996; Montello et al., 2003). This holds especially for natural regions or regions evolving from the social interactions of individual groups. Administrative regions, though, have well defined borders that have been fixed by an administrative body. Further, administrative regions may be identifiable by signage in the environment, for example, districts in a city may be labeled on street signs. But also some natural regions may be well usable for embedding a route in its environment, for example, clearly visible features such as large parks or water bodies.

These kinds of regions are integrated in the information displayed on RAMs in order to provide a global spatial context for wayfinding. In case they get lost, i.e., deviate from the route, wayfinders can navigate along regions to the region that contains the destination. In the following, we will explicate how the relevant regions can be identified automatically. Figure 7 shows the hierarchy of regions used in our approach. The top level regions we consider are those of country borders, followed by those of states within a country, cities and towns (or in general built-up areas). Within cities, we consider local particularities, namely districts and natural features. These features may be located within several regions, for example, the border between two districts may run through the middle of a park. We treat all regions within a city to be on the same level of granularity. 


\section{SCHMID, RICHTER, PETERS}

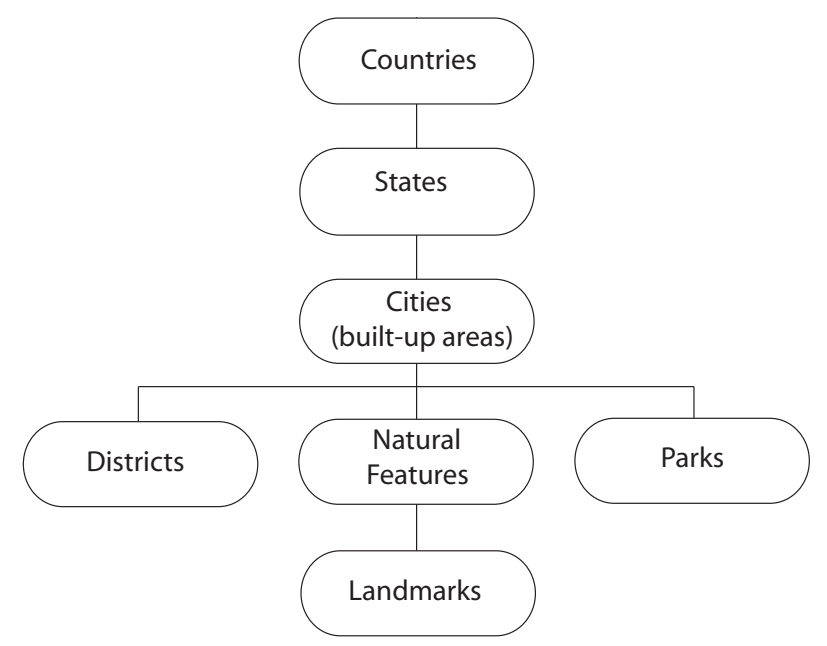

Figure 7.: The region hierarchy as implemented in the RAM process.

Selecting these regions for integration in RAMs is a pragmatic approach in terms of perceptibility, available data and computational efficiency. The algorithm for determining the regions to be included is illustrated next.

\section{Pre-Processing and Query Optimization}

The aim of the region extraction algorithm is to identify all regions that are relevant for the wayfinding problem. Relevant regions are those that contain parts of the routes-be it the main route or one of the alternative routes. These are the regions the wayfinders will pass through. The computation of the membership of a route within a region is based on the points contained in the route. If a point of the route is within a region, the region is considered relevant for the route. In terms of geographical data, regions are usually complex polygons (in our data some of the districts have up to 5000 geometric control points). Thus, depending on the type of regions and the length of the route, the number of regions and points to be checked can become very large, turning this into a computationally infeasible approach.

Therefore, to drive computation, initially we need to determine the region which completely contains the route. A route between Bremen and Hamburg would be fully contained in the region "Germany;" a route within Bremen would be completely contained within the region "Bremen." This region is the starting point for a further refinement of the relevant regions. The encompassing region serves two purposes: 1) only those regions that belong to a category on lower levels of the hierarchy than the encompassing region (see Fig. 7) will be relevant for the routes, 
i.e., we can restrict our search to these levels; 2) in a similar line of argument, we can restrict the search for regions to the spatial extend covered by the encompassing region. We will treat each hierarchy level relevant for the region identification individually.

The key idea of processing all regions that may be relevant for the RAM to be constructed is the separation of their functional and geometric representations as well as the utilization of the neighborhood relation between regions. To this end, we need to do some preprocessing on the region data. For each region, we compute their bounding box as this allows for a faster comparison of spatial relations between regions. Namely, we need to test for intersection/touch and containment of regions. Using the bounding boxes, we calculate an adjacency matrix that stores intersection and containment of regions-a region containing another is taken to intersect with the contained region.

With this adjacency matrix, we can now navigate efficiently through the hierarchy of regions, i.e., our search space. We further restrict the containment check of route in regions by only checking for decision points, i.e., those points of a route that coincide with an intersection. As stated before, region selection is performed on each layer of the hierarchy individually. However, by means of the indexed data, we can interlink the different layers to further facilitate efficient processing of the information. The following algorithm selects relevant regions for the information so far contained in the route aware map, namely the main route, the street network around origin and destination, and the alternative routes (see Fig. 8).

1. We compute the bounding box of the RAM (the extend covered by main route, origin and destination, ARs).

2. We determine the region on the lowest possible level of the hierarchy that fully contains the bounding box of the route aware map.

3. For every route (main route and ARs) we iterate through all nodes that correspond to decision points.

4. We check for containment within any of the regions of the current level in the hierarchy. In the first pass, this is the level one below the level the encompassing region belongs to.

- If no region contains a node of the route (not all region layers will cover the complete area as administrative regions do) we stop the search on this level and proceed with the next region layer at step 4 .

5. If we have identified a region whose bounding box contains a node, we check again with the actual border of the region. Nodes being on the border of a region count as being contained as well.

(a) If the node is really contained, we select the region and add it to the map. 
16 SCHMID, RICHTER, PETERS

(b) If the node is not contained, we select all neighbors of the selected region by means of the adjacency matrix. We check these neighbors for which of them contains the node.

6. If we have processed all nodes and there are still layers of the hierarchy we have not checked yet, we select one of these as new layer and repeat the process from step 3 on.

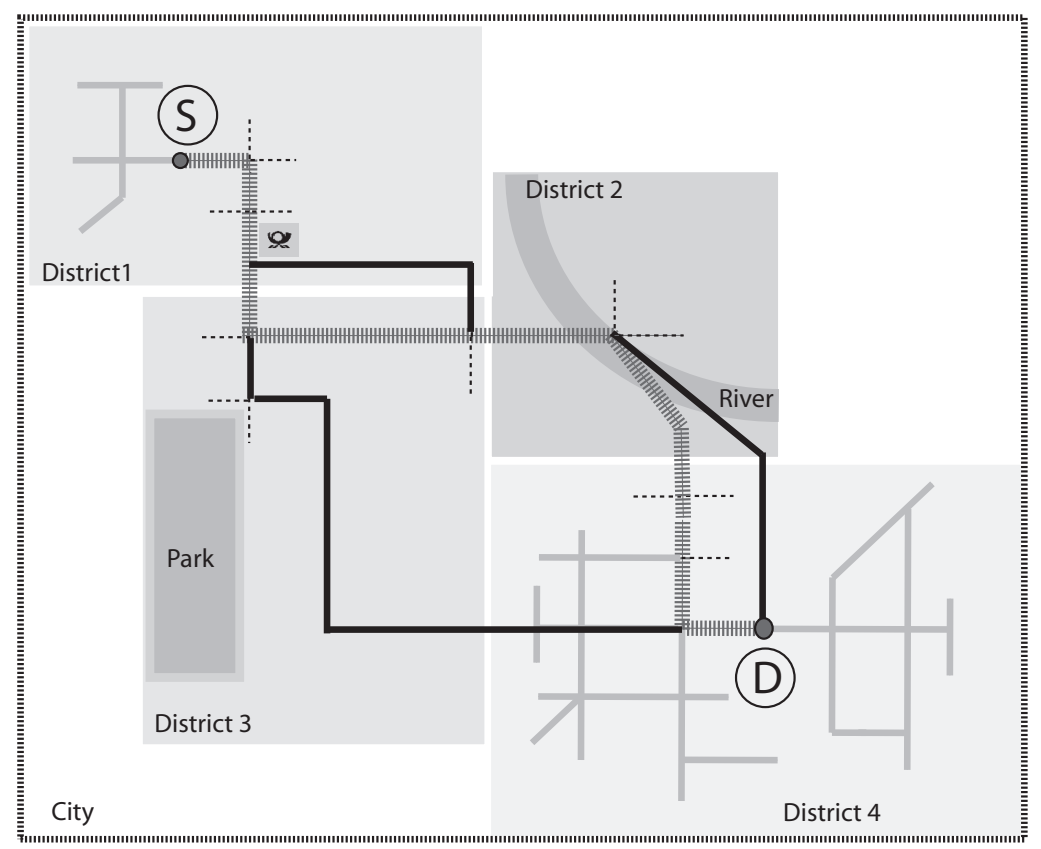

Figure 8.: Global orientation: Embedding the route in surrounding regions.

\subsubsection{Local Orientation: Landmarks}

Finally, in creating RAMs local landmarks are used to anchor route information in its surrounding environment. These landmarks serve two purposes: they help to disambiguate intersections and they foster orientation of wayfinders. In areas that are made up of structurally similar intersections, i.e., where the street network is very regular (see Section 3.2.2) landmarks may make the difference it takes to identify the intended intersection. Accordingly, for intersections identified to be structurally similar to their surrounding intersections, we check whether some landmarks may be used to identify them. This check is done as explained in our previous research (e.g., Richter \& Klippel, 2007; Richter, 2007), but extended 
ROUTE AWARE MAPS

to include depictional considerations. Additionally to ambiguity checks we have used in our previous work, we also check whether potential landmarks would be depicted on the map using the same cartographic style as other features in the local surroundings, i.e., whether the feature categories they belong to are mapped using the same color and shape. If two different types of features are depicted the same way (e.g., a fountain and a statue may both be depicted as a grey circle), they are not easily distinguished on the map and, therefore, do not unambiguously identify an intersection if both features are present in the surrounding area. In case a landmark located at the intended intersection unambiguously discriminates this intersection from its neighboring ones, it is included in the route aware map and, thus, solves a potential conflict.

Local landmarks may also be used to foster orientation, similar to global ones, i.e., those environmental features represented as regions. To this end, it is checked whether highly salient landmarks are located along the route (such as highly visible shops or monument buildings); these are added to the RAMs. However, to avoid visual clutter, we limit the number of landmarks added this way to only a few key features. Checking for such landmarks, again, is based on previous work.

\subsection{Example}

Figures 9 and 10 illustrate the steps of compiling a RAM based on geographical data of the city of Bremen. Figure 9a depicts the main route from origin $\mathrm{S}$ to destination D running through Bremen's inner city. Figure 9b shows the extracted strip map, the minimalist map-based representation of the route. In Figure 9c we disambiguate the start environment by extending the street network until the first intersection as described in Section 3.1. In the same step, we extend the street network around the destination (network depth of three topological nodes) and added the river as an additional landmark according to Section 3.3.2.

Figure 10a depicts the potentially problematic parts of the route and the resulting alternative routes (bold dark gray underlying lines). Figure 10b depicts the so so far compiled route (extended start and destination environments) with the extracted alternatives routes. Finally, in Figure 10c we add those regions which are relevant to the route (here, these are only districts as the route is contained in a single city). This results in the route aware map assisting a wayfinder to get from origin $\mathrm{S}$ to destination $\mathrm{D}$.

Note that we do not display cartographic labels, such as names of streets or districts, to emphasis the principle generation process of RAMs. For actual usage, without this information a real anchoring of the streets, regions, and landmarks in the global context is likely not possible. 


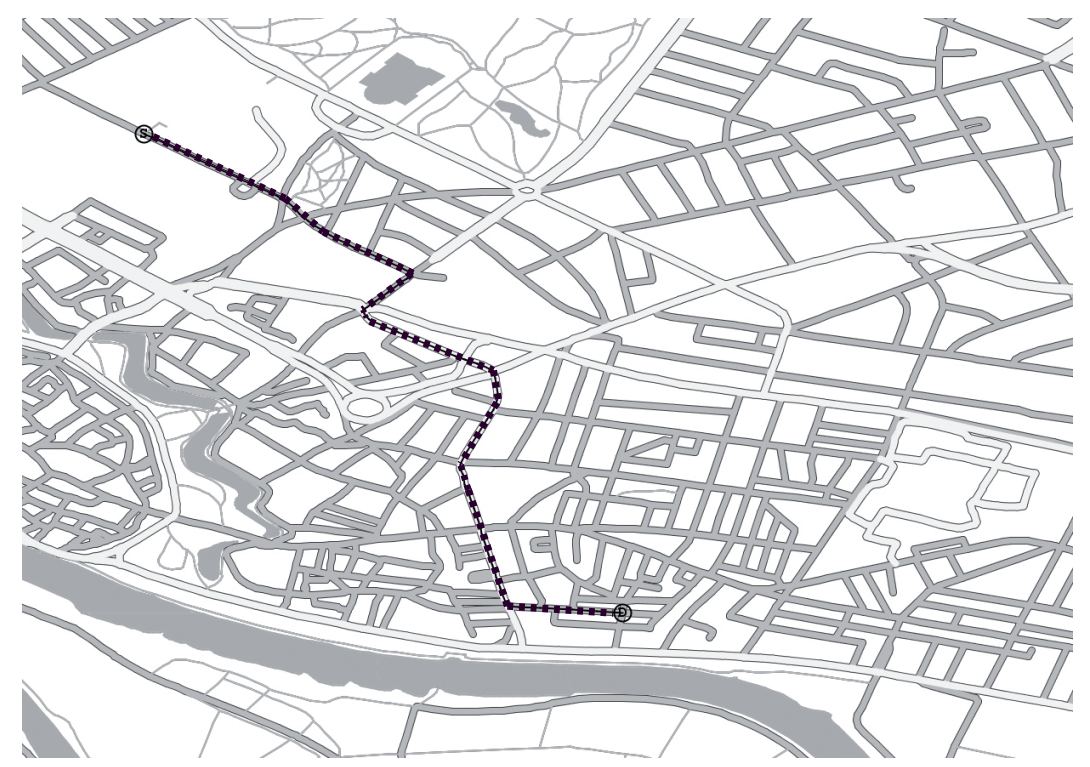

a)

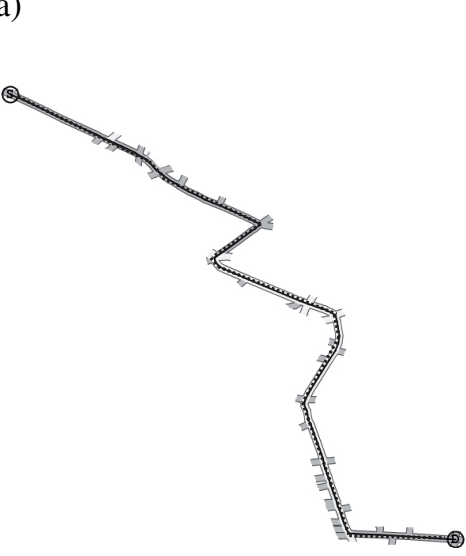

b)

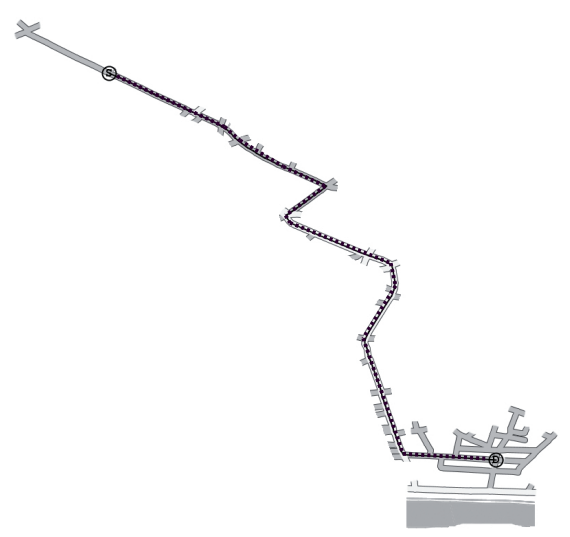

c)

Figure 9.: a) shows the original (survey) map with the route (thin black line) from $S$ to $D$. b) shows the extracted route, c) the route with disambiguated start environment and extended destination environment. 


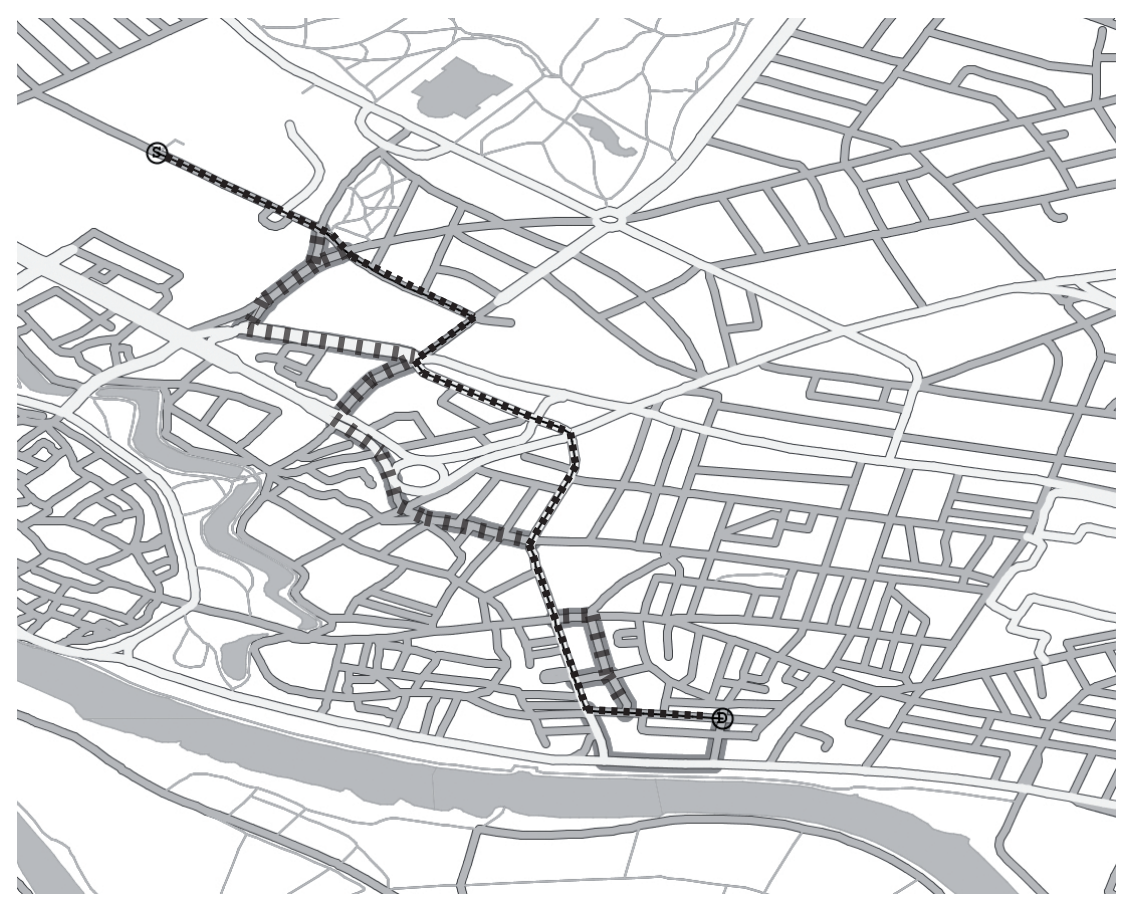

a)
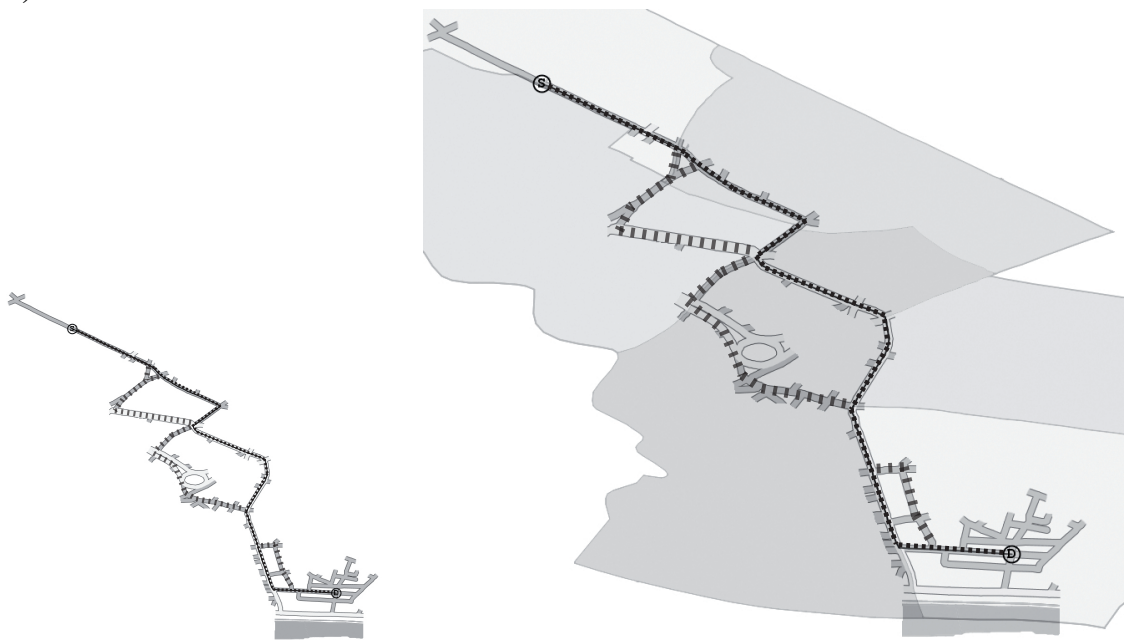

b)

c)

Figure 10.: a) shows the route in the original (survey) map with alternative routes (bold underlying lines). b) shows the extracted alternative routes, and c) depicts the final RAM with the relevant regions (in this case districts) for the main route and the alternative routes. 


\section{SCHMID, RICHTER, PETERS}

\section{Discussion}

Schematic wayfinding maps are by definition task specific representations (cf. Klippel et al., 2005) usually incorporating a stringent feature selection. The selection of features aims at efficient assistance with low cognitive demands tailored to a specific wayfinding task. There are different approaches to define, select, and visualize the relevant information for a given route underlying the specific wayfinding task, which leads to different schematization principles. In Agrawala $\&$ Stolte (2001), for example, the authors propose LineDrive Maps, which are based on the mental prototypical arrangements of intersections and activity based scaling of street segments. The resulting schematic maps represent a route as strip map with no further information on the surrounding environment and can be considered to be a data-driven schematization. As a contrast to this reduced representation, Focus Maps (Richter et al., 2008) use a rich representation of the environment and highlight the crucial elements (e.g., the route) by de-emphasizing non-relevant areas of the environment. This is achieved by a fading out of colors and a simplification of feature geometry based on proximity to the elements in focus. Schematization in Focus Maps is based on perceptual effects as the selection of features is mostly done via their color encoding. $\mu$ Maps (Schmid, 2008) show only the unfamiliar parts of a route in full detail, the familiar parts are schematized to a high degree. This kind of schematization exploits individual familiarity with an environment.

RAMs introduce a new type of schematization by taking these approaches a step further. They provide a multi-granular schematization since they select features of an environment on different levels of granularity. In that, they acknowledge the hierarchical representation of space by humans (e.g., Hirtle \& Heidorn, 1993). They provide means to reach the overall goal—getting to the destinationand at the same time offer information for reaching this goal on different levels of granularity, thus offering alternatives and allow for approximate wayfinding in case task execution fails. Thus, while being task-specific, they allow for different executions of the task.

RAMs communicate survey knowledge dependent on the course of a particular route. They do not show the details of the street network, especially of remote locations in the same district, however, they communicate the configurations of important structural and conceptual elements of the environment in relation to the actual route. They are rather conceptual survey maps than general purpose maps. RAMs illustrate the structure of the environment for a route similarly to how a GPS-based wayfinding assistance system only communicates the route to travel. They focus on the crucial parts of survey information. General purpose survey maps on the other hand offer all information of a defined region as equally important.

However, the selection of, for example, regions also depends on the network incorporated by the route. If a route incorporates a longer part across a highway, 
ROUTE AWARE MAPS 21

the context of that part is different to the context of a part of a route within an inner city environment. While driving along a highway, we are most likely not interested in every district of a city we pass or potentially cross. The selection of the contextual information for such routes would benefit from additional considerations of coherent parts of the route with respect to the utilized transportation link. Thus, in generating RAMs, the (conceptual) scale of covered route segments should be considered as well and the construction process should be accordingly adapted - this might include activity-based scaling similar to Agrawala \& Stolte (2001).

\section{Conclusions}

In this paper, we have presented an approach to map-based wayfinding assistance that we term route aware maps. These maps are designed to present all information that is needed to successfully reach a destination while aiming at sparseness in the display of that information. It combines the best of strip maps, which only present information on the route itself, with survey maps, which distribute information uniformly across the chosen area.

Next to the actual route from origin to destination, RAMs present the area around origin and destination in more detail to keep the wayfinders oriented at these crucial spots. The maps also integrate alternative routes at those points along the route where wayfinding errors likely occur due to the (local or global) ambiguity in the structure of the environment. Furthermore, RAMs embed the route in its global spatial context. To this end, those regions relevant for the route are identified and displayed allowing for approximate navigation using region information in case the route has been accidentally left; key local landmarks are shown as well and further the anchoring of the route within the environment. We take an information- and representation-theoretic approach to identifying this additional information that is required for successful navigation. We have detailed the construction process for route aware maps, stating algorithms for all intermediate steps, and discussed an example map.

We believe that route aware maps as they have been presented in this paper are a promising approach for solving two (related) problems in map-based navigation assistance: 1) provision of focused, easy to access assistance that still allows for error recovery; 2) the key-hole problem. The latter describes the problem of presenting local information in its global context such that users can easily relate both; it is especially crucial for the small displays of mobile devices. Thus, RAMs are not only more reliable in a given wayfinding situation, they may also help to overcome a major problem of today's assistance systems, namely that users do not really understand the spatial situation they are in and hardly remember anything of the route after reaching the destination as shown, for example, by Parush et al. (2007) and Ishikawa et al. (2008). 


\section{SCHMID, RICHTER, PETERS}

In the near future, we plan to test the performance of RAMs in empirical wayfinding studies in which we will compare the wayfinding performance of human subjects using route aware maps in real world navigation tasks with participants using 'classical' street-maps. We will also explore scale-dependent construction of RAMs and check whether additional salient objects should be included in the maps, for example, major roads that may provide additional global orientation, such as highways.

\section{Acknowledgments}

This work has been supported by the Transregional Collaborative Research Center SFB/TR 8 Spatial Cognition, which is funded by the Deutsche Forschungsgemeinschaft (DFG). We like to thank the participants of the 'You-Are-Here Maps' workshop at Spatial Cognition 2008 and three anonymous reviewers for their valuable feedback on our work.

\section{References}

Agrawala, M., \& Stolte, C. (2001). Rendering effective route maps: Improving usability through generalization. In SIGGRAPH 2001, (pp. 241-249). Los Angeles, California, USA: ACM Press.

Baus, J., Krüger, A., \& Wahlster, W. (2002). A resource-adaptive mobile navigation system. In IUI '02: Proceedings of the 7th international conference on Intelligent user interfaces, (pp. 15-22). New York, NY, USA: ACM Press.

Couclelis, H. (1996). A typology of geographic entities with ill-defined boundaries. In P. A. Burrough, \& A. U. Frank (Eds.) Geographic Objects with Indeterminate Boundaries, (pp. (45-55). London: Taylor \& Francis.

Daniel, M.-P., \& Denis, M. (1998). Spatial descriptions as navigational aids: A cognitive analysis of route directions. Kognitionswissenschaft, 7, 45-52.

Dijkstra, E. (1959). A note on two problems in connexion with graphs. $\mathrm{Nu}$ merische Mathematik, 1, 269-271.

Duckham, M., \& Kulik, L. (2003). "Simplest" paths: Automated route selection for navigation. In W. Kuhn, M. Worboys, \& S. Timpf (Eds.) Spatial Information Theory. Foundations of Geographic Information Science, LNCS 2825, (pp. 169-185). Berlin: Springer. International Conference COSIT.

Freksa, C. (1999). Spatial aspects of task-specific wayfinding maps - a representation-theoretic perspective. In J. S. Gero, \& B. Tversky (Eds.) Visual and Spatial Reasoning in Design, (pp. 15-32). University of Sidney. 
Golledge, R. G. (1999). Wayfinding Behavior - Cognitive Mapping and Other Spatial Processes. Baltimore, USA: John Hopkins University Press.

Haque, S., Kulik, L., \& Klippel, A. (2007). Algorithms for reliable navigation and wayfinding. In T. Barkowsky, M. Knauff, G. Ligozat, \& D. R. Montello (Eds.) Spatial Cognition V - Reasoning, Action, Interaction, LNCS4387, (pp. 308-326). Berlin: Springer.

Hirtle, S. C., \& Heidorn, P. B. (1993). The structure of cognitive maps: Representations and processes. In T. Gärling, \& R. Golledge (Eds.) Behaviour and Environment: Psychological and Geographical Approaches, chap. 7, (pp. 170192). Amsterdam, North-Holland: Elsevier Science Publishers.

Ishikawa, T., Fujiwara, H., Imai, O., \& Okabe, A. (2008). Wayfinding with a GPS-based mobile navigation system: A comparison with maps and direct experience. Journal of Environmental Psychology, 28, 74-82.

Klippel, A. (2003). Wayfinding choremes. In W. Kuhn, M. Worboys, \& S. Timpf (Eds.) Spatial Information Theory. Foundations of Geographic Information Science, LNCS 2825, (pp. 320-334). Berlin: Springer. International Conference COSIT.

Klippel, A., \& Montello, D. R. (2007). Linguistic and nonlinguistic turn direction concepts. In S. Winter, M. Duckham, L. Kulik, \& B. Kuipers (Eds.) Spatial Information Theory, LNCS 4736, (pp. 354-372). Berlin: Springer. International Conference COSIT.

Klippel, A., Richter, K.-F., Barkowsky, T., \& Freksa, C. (2005). The cognitive reality of schematic maps. In L. Meng, A. Zipf, \& T. Reichenbacher (Eds.) Map-based Mobile Services - Theories, Methods and Implementations, (pp. 5774). Berlin: Springer.

Lynch, K. (1960). The Image of the city. MIT Press.

MacEachren, A. M. (1995). How maps work: representation, visualization, and design. Guilford Press; New York.

Michon, P.-E., \& Denis, M. (2001). When and why are visual landmarks used in giving directions? In D. R. Montello (Ed.) Spatial Information Theory. Cognitive and Computational Foundations of Geopraphic Information Science, LNCS 2205, (pp. 400-414). Berlin: Springer. International Conference COSIT.

Montello, D., Goodchild, M., Gottsegen, J., \& Fohl, P. (2003). Where's downtown? behavioral methods for determining referents of vague spatial queries. Spatial Cognition and Computation, 3(2), 185-204. 


\section{SCHMID, RICHTER, PETERS}

Montello, D. R. (2005). Navigation. In P. Shah, \& A. Miyake (Eds.) Handbook of Visuospatial Thinking, (pp. 257-294). Cambridge: Cambridge University Press.

Parush, A., Ahuvia, S., \& Erev, I. (2007). Degradation in spatial knowledge acquisition when using automatic navigation systems. In S. Winter, M. Duckham, L. Kulik, \& B. Kuipers (Eds.) Spatial Information Theory, LNCS 4736, (pp. 238-254). Berlin: Springer.

Phillips, R. J. (1979). Making maps easy to read: A summary of research. In P. A. Koler, M. E. Wrolstad, \& H. Bouma (Eds.) Processing of Visual Language, vol. 1, (pp. 165-174). New York: Plenum Press.

Richter, K.-F. (2007). A uniform handling of different landmark types in route directions. In S. Winter, M. Duckham, L. Kulik, \& B. Kuipers (Eds.) Spatial Information Theory, LNCS 4736, (pp. 373-389). Springer; Berlin. International Conference COSIT.

Richter, K.-F., \& Duckham, M. (2008). Simplest instructions: Finding easy-todescribe routes for navigation. In T. J. Cova, H. J. Miller, K. Beard, A. U. Frank, \& M. F. Goodchild (Eds.) Geographic Information Science - 5th International Conference, GIScience 2008, LNCS 5266, (pp. 274-289). Springer; Berlin.

Richter, K.-F., \& Klippel, A. (2005). A model for context-specific route directions. In C. Freksa, M. Knauff, B. Krieg-Brückner, B. Nebel, \& T. Barkowsky (Eds.) Spatial Cognition IV. Reasoning, Action, Interaction: International Conference Spatial Cognition 2004, LNAI 3343, (pp. 58-78). Berlin: Springer.

Richter, K.-F., \& Klippel, A. (2007). Before or after: Prepositions in spatially constrained systems. In T. Barkowsky, M. Knauff, G. Ligozat, \& D. R. Montello (Eds.) Spatial Cognition V - Reasoning, Action, Interaction, LNAI 4387, (pp. 453-469). Berlin: Springer.

Richter, K.-F., Peters, D., Kuhnmünch, G., \& Schmid, F. (2008). What do focus maps focus on? In C. Freksa, N. Newcombe, P. Gärdenfors, \& S. Wölfl (Eds.) Spatial Cognition VI - Learning, Reasoning, and Talking about Space, LNAI 5248, (pp. 154-170). Berlin: Springer.

Rosenholtz, R., Li, Y., \& Nakano, L. (2007). Measuring visual clutter. Journal of Vision, 7(2), 1-22.

Ross, T., May, A., \& Thompson, S. (2004). The use of landmarks in pedestrian navigation instructions and the effects of context. In S. Brewster, \& M. Dunlop (Eds.) Mobile Computer Interaction - MobileHCI 2004, LNCS 3160, (pp. 300304). Berlin: Springer. 
Schmid, F. (2008). Knowledge based wayfinding maps for small display cartography. Journal of Location Based Services, 2(1), 57-83.

Schneider, L. F., \& Taylor, H. A. (1999). How to get there from here? Mental representations of route descriptions. Applied Cognitive Psychology, 13, 415441.

Seifert, I., Barkowsky, T., \& Freksa, C. (2007). Region-based representation for assistance with spatio-temporal planning in unfamiliar environments. In G. Gartner, W. Cartwright, \& P. Peterson, M. (Eds.) Location Based Services and TeleCartography, (pp. 179-191). Berlin: Springer.

Tversky, B., \& Lee, P. U. (1998). How space structures language. In C. Freksa, C. Habel, \& K. F. Wender (Eds.) Spatial Cognition: An Interdisciplinary Approach to Representing and Processing Spatial Knowledge, (pp. 157-175). Berlin: Springer. LNAI 1404.

Tversky, B., \& Lee, P. U. (1999). Pictorial and verbal tools for conveying routes. In C. Freksa, \& D. M. Mark (Eds.) Spatial Information Theory, (pp. 51-64). Berlin: Springer.

Wiener, J., \& Mallot, H. (2003). 'Fine-to-coarse' route planning and navigation in regionalized environments. Spatial Cognition and Computation, 3(4), 331-358. 